\title{
Efficient operator splitting for modelling transport and transformations of multiple nitrogen species in a variably-saturated subsurface environment
}

\author{
S.J.R. Woodward $^{\text {a }}$ and F. Wang ${ }^{b}$ \\ ${ }^{a}$ Lincoln Ventures Limited, Private Bag 3062, Hamilton, New Zealand \\ ${ }^{b}$ Fortescue Metals Group Ltd, Level 2, 87 Adelaide Terrace, East Perth, WA 6004, Australia \\ Email: simon.woodward@,lvl.co.nz
}

\begin{abstract}
In order to study the potential impacts of nitrogen leaching from agricultural land into the vadose zone and shallow groundwater systems that drain into New Zealand's oligotrophic lakes, we coupled a multi-species reaction model with an existing 3D variably-saturated finite element flow and transport model, FEMWATER (Lin et al., 1997). The resulting code is called FEMWATER-N (Wang et al., 2003).

FEMWATER solves the transient advection-dispersion partial differential equation (PDE) using a Lagrangian-Eulerian method. In FEMWATER-N this transport solver was coupled with a reaction ordinary differential equation (ODE) system solver using an operator splitting approach. Initially the reaction ODE was solved using an iterative exponential Euler method, and the coupled transport-reaction PDE-ODE system was solved using standard sequential iterative operator splitting.

Improvements to FEMWATER-N described in this paper include implementing the popular LSODE (Livermore solver for ODEs) code to solve the non-linear reaction ODE system at every mesh node, and a symmetric iterative operator splitting method to solve the coupled advection-dispersion-reaction PDE-ODE system.

Solution time was dominated by calls to the transport solver. The symmetric operator split allowed more rapid convergence of the coupled solvers, and, by preconditioning the operator split with an additional reaction call, a single iteration was sufficient to obtain an accurate solution to the coupled equations after the first few time steps. This approach achieved a $47 \%$ reduction in CPU time in our example. The symmetric operator splitting method is also free from mass balance error.
\end{abstract}

Keywords: Reactive transport model, vadose zone, groundwater, finite element method, nitrate 


\section{INTRODUCTION}

Agricultural-source nitrate discharging to surface waters is a major environmental problem in New Zealand, and has been identified as the primary cause of eutrophication in several iconic lakes and rivers, including Lake Taupo, the largest lake in Australasia. Several years of groundwater monitoring in a small volcanic hill catchment at Waihora on the northern shores of Lake Taupo has shown that while shallow groundwater is typically high in nitrate, deeper groundwater may be relatively nitrate-free, indicating the occurrence of heterotrophic denitrification in the subsurface (Stenger et al., 2010). We sought to develop a numerical model to understand the nitrogen transport, transformations, and discharge relating to the groundwater observation at the Waihora site, and to assess potential nitrate assimilation and discharge under future land use scenarios.

There are a number of groundwater models that estimate natural attenuation of contaminants (Mulligan and Yong, 2004), or denitrification in particular (Lee et al., 2009). However, there seem to be no denitrification models designed to consider attenuation in the vadose zone or in complex topographies, which are important considerations for nitrate leaching from grazed pastures located on rolling or steep terrain.

We therefore modified the variably saturated, finite element flow and transport model FEMWATER (Lin et al., 1997) to simulate movement and transformations of nitrogen (N) species leached from agricultural land through the vadose and groundwater zones. The modified model is named FEMWATER-N and has been described by Wang et al. (2003) and Woodward et al. (2009).

The chemical kinetics in FEMWATER-N were based on the model of Kinzelbach et al. (1991). The Kinzelbach model described interactions between oxygen, nitrate, organic carbon substrate and bacteria, due to aerobic decomposition and denitrification processes. Bacteria were assumed to be immobile (adhered to soil particles), but chemical species were present in three different phases (dissolved, microbial/bio, and adsorbed). Species transformations took place in the microbial/bio phase. FEMWATER-N added organic-N and ammonium-N as inputs, and mineralisation and nitrification processes, while considering microbial biomass to be constant/ubiquitous. Figure 1 summarises the chemical species, phases and transformations modelled in FEMWATER-N.

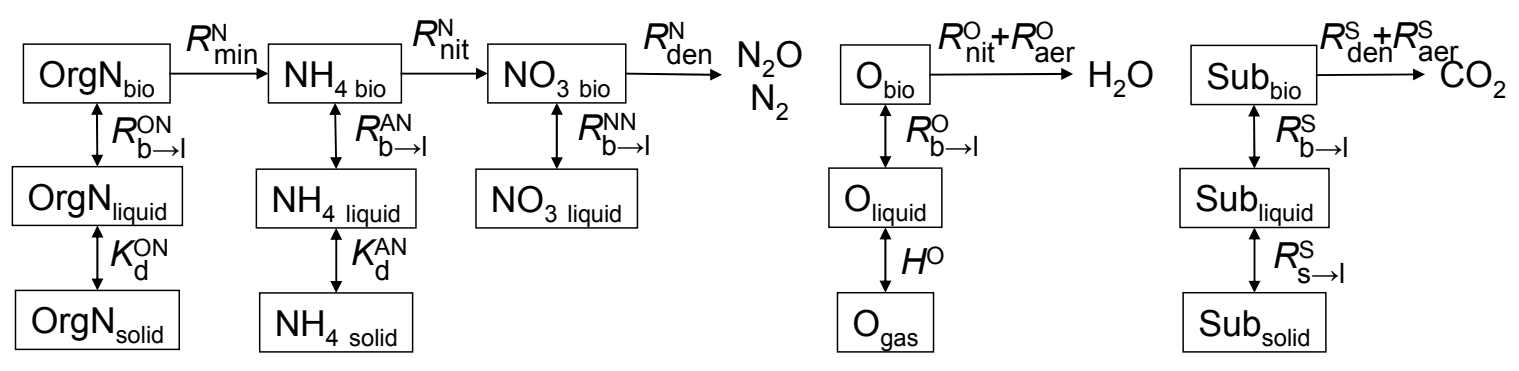

Figure 1: Chemical species, phases and transformations.

FEMWATER-N has been used previously for several point source studies, including simulating discharge from a wastewater treatment plant as described in Wang et al. (2003), and discharge from septic tanks and two other wastewater treatments plants (Barkle and Wang, 2001, 2003, 2005; Barkle, 2004). In these studies FEMWATER-N predictions of contamination and attenuation were broadly consistent with well samples, although the model has yet to be comprehensively calibrated or validated (Barkle and Wang, 2003, p.59). Basic sensitivity analysis was carried out for several model input parameters.

When we set up FEMWATER-N to simulate the current land use at the Waihora site, we encountered numerical overflow, slow- and non-convergence, and negative concentration issues with the solution of the reaction equations, which formed a stiff system. The analysis and reworking of the transport-reaction operator splitting method described in this paper was carried out in response to these problems. These improvements were necessary to allow reliable analysis of nitrate assimilation and discharge under alternative future land use scenarios within a reasonable timeframe.

\section{FLOW AND TRANSPORT EQUATIONS}

The full set of equations describing water and solute flows and transformations in FEMWATER-N form a coupled non-linear PDE-ODE system.

The density-dependent Richards equation for flow in Cartesian coordinates is given in Lin et al. (1997) as: 


$$
\begin{aligned}
& \frac{\rho}{\rho_{0}} F \frac{\partial h}{\partial t}=\nabla \cdot\left[K \cdot\left(\nabla h+\frac{\rho}{\rho_{0}} \nabla z\right)\right]-\frac{\rho}{\rho_{0}} q \\
& F=\alpha^{\prime} \frac{\theta}{n}+\beta^{\prime} \theta+n \frac{d \theta_{e}}{d h}
\end{aligned}
$$

where $\rho$ is the water density, $\rho_{0}$ is the reference water density at zero concentration, $F$ is the storage coefficient, $h$ is the pressure head, $t$ is time, $K$ is the hydraulic conductivity tensor (which is a non-linear function of $h$ ), $z$ is elevation, $q$ represents source and sink terms, $\alpha^{\prime}$ and $\beta$ are the dimensionless modified compressibilites of the medium and of water respectively, $\theta$ is moisture content, $n$ is effective porosity, and $\theta_{\mathrm{e}}$ is the saturation. Dirichlet, Cauchy, Neumann and Variable Flux boundary condition options are provided. FEMWATER uses the Galerkin finite element method in space, and the finite difference method in time, to formulate the resulting steady-state or transient flow problem. The matrix equation, which is highly nonlinear in the unsaturated zone, is solved using a conjugate gradient solver with Picard iteration and dynamic relaxation.

In the general case of density dependent flow, fluid density $\rho$ is a function of solute concentration $C$, and (1) is coupled with the advection-dispersion transport equation (Lin et al., 1997):

$$
\begin{aligned}
& \left(\theta \frac{\partial C}{\partial t}+\rho_{b} \frac{\partial C_{S}}{\partial t}\right)+V \cdot \nabla C-\nabla \cdot(\theta D \cdot \nabla C)= \\
& -\alpha^{\prime} \frac{\partial h}{\partial t}\left(\theta C+\rho_{b} C_{S}\right)-q C+\left(F \frac{\partial h}{\partial t}+\frac{\rho_{0}}{\rho} V \cdot \nabla\left(\frac{\rho}{\rho_{0}}\right)-\frac{\partial \theta}{\partial t}\right) C+R
\end{aligned}
$$

where $C$ is the dissolved concentration, $\rho_{\mathrm{b}}$ is the bulk density of the medium, $C_{\mathrm{S}}$ is the adsorbed concentration, $V$ is the Darcy flux, $D$ is the dispersion coefficient tensor, and $R$ represents "reaction source" terms in FEMWATER-N. Again, Dirichlet, Cauchy, Neumann and Variable Flux boundary condition options are provided. FEMWATER solves this equation using the modified method of characteristics (MMOC, Zhang et al., 1993) for the advection term, and the weighted residual finite element method for the dispersion term. Again, the finite difference method is used in time, and the matrix equations are solved using a conjugate gradient solver with Picard iteration.

If chemical concentrations are low, as in our application, density dependence is negligible, and (1) and (3) are no longer coupled.

\section{REACTION EQUATIONS}

The $R$ term on the right hand side of (3) represents either first-order phase transfer between the liquid and bio phases and/or first-order kinetic sorption of substrate to solid phase in FEMWATER-N, as shown in Figure 1. Organic-N and ammonium-N were modelled as having equilibrium-controlled sorption, and oxygen was modelled as having a gas phase maintained in equilibrium with the dissolved phase using a Henry's law constant $H^{\mathrm{O}}$. Multiple Monod kinetics were used for mineralization, nitrification, aerobic decomposition, and denitrification in the bio phase. The reaction equations are detailed in Wang et al. (2003), and an errata is provided in the Appendix of the current paper. As an example, the equations for organic-N (ON) were,

$$
\begin{aligned}
& \frac{\partial\left(\theta C^{O N}+\rho_{b} C_{s}^{O N}\right)}{\partial t}=L\left(C^{O N}\right)+R_{b \rightarrow l}^{O N} \\
& \frac{\partial\left(\theta_{b} C_{b}^{O N}\right)}{\partial t}=-R_{b \rightarrow l}^{O N}-R_{\min }^{N} \\
& C_{s}^{O N}=K_{d}^{O N} C^{O N}
\end{aligned}
$$

where $C^{\mathrm{ON}}, C_{\mathrm{s}}^{\mathrm{ON}}$ and $C_{\mathrm{b}}^{\mathrm{ON}}$ are the concentrations in liquid, solid and bio phase respectively, $L$ is the transport operator specified by (3) $\theta_{\mathrm{b}}$ is the bio phase volume, and $K_{\mathrm{d}}^{\mathrm{ON}}$ is the distribution coefficient. The reaction rates $R_{b \rightarrow l}^{O N}$ and $R_{\min }^{N}$ are the kinetic bio to liquid phase exchange rate, and the (multiple Monod) mineralization rate respectively: 


$$
\begin{aligned}
& R_{b \rightarrow l}^{O N}=\alpha^{O N} \theta_{b}\left(C_{b}^{O N}-K_{b \rightarrow l}^{O N} C^{O N}\right) \\
& R_{\min }^{N}=V_{\min }^{\max } \theta_{b}\left(1-f_{O}\right) \frac{C_{b}^{O N}}{K_{\min }^{O N}+C_{b}^{O N}} \frac{C_{b}^{O}}{K_{\min }^{O}+C_{b}^{O}} \\
& f_{O}=\frac{1}{2}-\frac{1}{\pi} \arctan \left(f_{s l}\left(C_{b}^{O}-O_{t}\right)\right)
\end{aligned}
$$

where $\alpha^{\mathrm{ON}}$ is the phase exchange rate, $K_{\mathrm{b} \rightarrow 1}^{\mathrm{ON}}$ is an equilibrium constant, $V_{\min }{ }^{\max }$ is the maximum mineralisation rate, $f_{\mathrm{O}}$ is a function that defines the switch from aerobic to anaerobic conditions, and $K_{\min }$ ON and $K_{\min }{ }^{\mathrm{O}}$ are Monod constants.

Similar equations govern the transformations of ammonium-N, dissolved oxygen, dissolved organic carbon substrate and nitrate-N. In reality the bio phase volume $\theta_{\mathrm{b}}$ is very small $\left(10^{-7}-10^{-5}\right.$, Widdowson, 1991$)$, so a nominal value of 1 is used to reduce the stiffness of the ODE system.

These chemical transformations are calculated independently at every mesh node in space, while the transport equation (3) calculates the movement of solutes in space.

Assuming a constant transport source $L$, the reaction equations form a system of coupled non-linear ordinary differential equations. Previously this system was solved using an iterated exponential Euler method. The more powerful Adams-Moulton method included with ODEPACK (Hindmarsh, 1983) has since been implemented to provide greater flexibility and better error control.

\section{OPERATOR SPLITTING}

The reaction equations were coupled to the original FEMWATER transport equations using the method of "operator splitting". In its simplest form, this involves solving the transport equations (3), and then using the result as the initial condition for solving the reaction equations (4), for each time step. Operator splitting is easy to implement, can be highly accurate, and allows the use of efficient specialised solvers to be used to solve the PDE and ODE portions of the model. Carrayrou et al. (2004) provide an excellent comparison of four common operator splitting approaches.

The FEMWATER-N reaction equations (4) were originally coupled with the FEMWATER transport model (3) using "standard sequential iterative" operator splitting (Yeh and Tripathi 1989; Carrayrou et al., 2004). In this approach, a constant "reaction source" term $R$ is added to the transport equation (3), whose value at each node is determined iteratively. The solution steps at each time step were as follows ( $X$ represents the five solutes):

1. Begin with the concentrations at the end of the previous time step, $C^{X}(t), C_{s}{ }^{X}(t)$ and $C_{b}{ }^{X}(t)$, and previous reaction source rates $R^{X}(t)$.

2. Assume $R^{X}(t+\Delta t)=R^{X}(t)$.

3. Solve transport PDE for each solute, using the latest reaction source rates $R^{X}(t+\Delta t)$, to get $C^{X}(t+\Delta t)$.

4. Solve reaction ODEs at each mesh node, using latest estimates of concentration $C^{x}(t+\Delta t)$ as initial condition, to get $C_{s}{ }^{X}(t+\Delta t)$ and $C_{b}{ }^{X}(t+\Delta t)$ and update $R^{X}(t+\Delta t)$.

5. Repeat steps 3-4 until $C^{x}(t+\Delta t)$ has converged.

While this standard sequential iterative method is perfectly serviceable, it is not mass conservative, and is not the most efficient in terms of the number of iterations required. For this reason, the operator splitting method in FEMWATER-N was upgraded to use the more efficient "symmetric sequential iterative" scheme (also described in Carrayrou et al., 2004). In this scheme, the transport equations are solved as before, assuming constant reaction source-sink terms. "Transport source" terms $L$ are then estimated by inverting (3):

$$
L(t+\Delta t)=\left(\theta+\rho_{b} K_{d}\right) \frac{C(t+\Delta t)-C(t)}{\Delta t}-R(t+\Delta t)
$$

These are treated as constant source terms in the reaction equations (3). The operator split method thus becomes:

1. Begin with the concentrations at the end of the previous time step, $C^{X}(t), C_{s}{ }^{X}(t)$ and $C_{b}{ }^{X}(t)$, and previous transport and reaction source rates $L^{X}(t)$ and $R^{X}(t)$.

2. Optional preconditioning step to estimate $R^{X}(t+\Delta t)$, described below.

3. Solve transport PDE for each solute, using the latest reaction source rates $R^{X}(t+\Delta t)$, to get $C^{X}(t+\Delta t)$ and update $L^{X}(t+\Delta t)$.

4. Solve reaction ODEs at each mesh node, using latest transport source rates $L^{x}(t+\Delta t)$, to get $C^{x}(t+\Delta t)$, $C_{s}^{X}(t+\Delta t), C_{b}{ }^{X}(t+\Delta t)$ and update $R^{X}(t+\Delta t)$. 
Woodward and Wang, Efficient operator splitting for modelling transport and transformations

5. Repeat steps 3-4 until $C^{X}(t+\Delta t), C_{s}^{X}(t+\Delta t)$, and $C_{b}{ }^{x}(t+\Delta t)$ have converged.

According to Carrayrou et al. (2004), symmetric sequential iterative schemes avoid additional mass balance error due to operator splitting.

\section{PRECONDITIONER}

With iterative schemes, a good initial estimate can improve how quickly the solution converges. Initially, the "reaction source" rates were carried over into the next time step for use as the initial estimate. This resulted in at least two iterations of the coupled transport-reaction solver in each time step. Including an additional initial reaction solver step (which was computationally much faster than the transport solver) to estimate reaction rates for the time step meant that a single iteration was often sufficient for convergence. As computation time was dominated by the number of calls to the transport solver, this resulted in a faster solution.

\section{RESULTS}

The methods were compared on a 30 day simulation (150 time steps) of the full reactive transport model of the Waihora research site, as described in Woodward et al. (2009). The modelled area is a hillslope pasture sloping down towards a wetland, and bounded by groundwater monitoring wells. The area is $83.1 \mathrm{~m}$ long, tapering from $60.6 \mathrm{~m}$ wide at the upper boundary to $33.5 \mathrm{~m}$ wide at the lower boundary, a total of $3914.7 \mathrm{~m}^{2}$. There are three material layers, representing a surface layer of Taupo ignimbrite, a thin layer of buried Palaesols, and a thick layer of Oruanui ignimbrite. Model depth decreased from $28.2 \mathrm{~m}$ at the upper boundary to $21.2 \mathrm{~m}$ at the lower boundary. The unstructured finite element mesh consisted of 23 layers of 635 triangular prism elements (a total of 14605 elements/8472 nodes). Upslope and downslope boundaries were constant head, while the side boundaries were aligned with assumed flow lines. Time steps were 0.1 day for the first 6 days, 0.2 days for the next 12 days, and 0.4 days for the last 12 days. A steady-state flow solution was used.

Because error control was managed by iterative operator splitting approaches, CPU time was used to compare the methods. The results are shown in Table 1. Total nitrate change was also recorded as a proxy for mass balance error (initial nitrate content was $10.493 \mathrm{~kg}$ in liquid and $18.545 \mathrm{~kg}$ in bio).

Table 1: Reaction-transport solver method benchmark results.

\begin{tabular}{|l|c|c|c|c|c|c|}
\hline Method & $\begin{array}{c}\text { Transport } \\
\text { Solver } \\
\text { Calls }\end{array}$ & $\begin{array}{c}\text { Reaction } \\
\text { Solver } \\
\text { Calls }\end{array}$ & $\begin{array}{c}\text { CPU } \\
\text { Time (s) }\end{array}$ & $\begin{array}{c}\text { Dissolved } \\
\text { NO } \text { Mass }_{\text {Mas }} \\
\text { Change } \\
\mathbf{( k g )}\end{array}$ & $\begin{array}{c}\text { Microbial } \\
\text { NO } \\
\text { Change } \\
\text { (kg) }\end{array}$ & $\begin{array}{c}\text { Denitrification } \\
\text { (kg) }\end{array}$ \\
\hline Exponential Euler & 379 & 379 & 751 & 5.5305 & 9.8085 & 14.594 \\
\hline + Preconditioner Step & 382 & 532 & 756 & 5.5307 & 9.8087 & 14.594 \\
\hline Adams-Moulton & & & & & & \\
\hline + Symmetric Splitting & 300 & 300 & 633 & 5.5233 & 9.8148 & 14.689 \\
\hline + Preconditioner Step & 159 & 309 & 398 & 5.5233 & 9.8148 & 14.689 \\
\hline
\end{tabular}

The numerical results produced by the different methods were similar, suggesting that mass balance errors were negligible. CPU time was primarily determined by the number of calls to the transport solver. The results suggested firstly that symmetric splitting allowed faster convergence of the transport-reaction solution, and secondly that an additional reaction step allowed further gains, but only in the case of the symmetric operator splitting. This was because, in the case of the standard sequential operator splitting method, the preconditioner calculation of $R$ was identical to the value used in the previous time step.

These methods were very easy to implement in FEMWATER-N, because transport only occurs in the liquid phase, and reactions in the bio phase, and resulted in greatly improved performance of the split operator solver, reducing CPU time by $47 \%$ in this example. Symmetric splitting by itself improved the solution speed notably, due to the improved convergence properties of the method, and addition of a preconditioner step in this case made a huge difference, reducing the number of transport solver calls to one in most time steps.

\section{CONCLUSIONS}

Since reactive-transport problems are generally non-linear, iterative methods are usually required. In particular, solution of the transport equation for each solute may be slow, so reducing the number of iterations is helpful. Symmetric sequential iterative splitting, with an additional reaction step as a preconditioner, was straightforward to implement and gave excellent results in our application. 
While FEMWATER is not the most modern code in terms of its solution methods and features, it provided the flexibility we needed to simulate distributed multispecies transport and reactions in a variably saturated, complex 3D domain. We were able to implement a customised reaction model and efficiently couple this with the existing transport solver, to develop a research tool for understanding nitrogen transformations and discharge relating to the groundwater observation at our Waihora research site. We are currently implementing an improved solute mass balance report for FEMWATER-N, which will allow us to generate land use impact estimates for this site, and for extension to similar hill farming landscapes that threaten the volcanic plateau lakes.

\section{ACKNOWLEDGMENTS}

This work was funded under the New Zealand Foundation for Research, Science and Technology "Groundwater Quality" (LVLX0302) and "Groundwater Assimilative Capacity" (C03X1001) contracts. Thanks to two anonymous referees, and to Channa Rajanayaka of Aqualinc Research Limited, whose comments were very helpful in improving this manuscript.

\section{REFERENCES}

Barkle, G., and Wang, F. (2001). Modelling the effects of seepage from dairy farm effluent treatment ponds on groundwater quality. Lincoln Environmental Research report number 2993, prepared for Environment Waikato.

Barkle, G., and Wang, F. (2003). The simulated fate of treated domestic effluent discharged into the vadose zone within the Taupo catchment. Lincoln Environmental Research report number 4949, prepared for Environment Waikato.

Barkle, G. (2004). Acacia Bay and Kinloch SBR groundwater models-comparison against monitoring data. Aqualinc report number HA03100/01.

Barkle, G., and Wang, F. (2005). Comparison of simulated to measured groundwater nitrate-N concentrations from SBR discharges in the Taupo catchment. Environment Waikato Technical Report 2006/32. Accessed at http://www.ew.govt.nz/Publications/Technical-Reports/

Carrayrou, J., Mosé, R., and Behra, P. (2004). Operator-splitting procedures for reactive transport and comparison of mass balance errors. Journal of Contaminant Hydrology, 68, 239-268.

Hindmarsh, A.C. (1983). ODEPACK, a systematized collection of ODE solvers. In: Stepleman, R.S., et al. (Eds.), Scientific Computing. North-Holland, Amsterdam. Pp. 55-64.

Kinzelbach, W., Schäfer, W., and Herzer, J. (1991). Numerical modeling of natural and enhanced denitrification processes in aquifers. Water Resources Research, 27, 1123-1135.

Lee, E.J., Kim, M., Kim, Y., and Lee, K.K. (2009). Numerical and field investigation of enhanced in situ denitrification in a shallow-zone well-to-well recirculation system. Ecological Modelling, 220, 2441-2449.

Lin, H.C., Richards, D.R., Talbot, C.A., Yeh, G.T., Cheng, J.R., Cheng, H.P., and Jones, N.L. (1997). FEMWATER: A three-dimensional finite element computer model for simulating density-dependent flow and transport in variably saturated media, Version 3.0. Report CHL-97-12, U.S. Army Corps of Engineers, 3909 Halls Ferry Road, Vicksburg, MS 39180-6199.

Mulligan, C.N., and Yong, R.N. (2004). Natural attenuation of contaminated soils. Environment International, 30, 587-601.

Severino, G., Monetti, V.M., Santini, A., and Toraldo, G. (2006). Unsaturated transport with linear kinetic sorption under unsteady vertical flow. Transport in Porous Media, 63, 147-174.

Stenger, R., Woodward, S.J.R., Clague, J., Wall, A., and Moorhead, B. (2010). How the spatial and temporal variation of reduced groundwater zones affects nitrate discharge into a wetland. In: The New Zealand Hydrological Society Conference, 7-10 December 2010, Dunedin, New Zealand.

Wang, F., Bright, J., and Hadfield, J. (2003). Simulating nitrate transport in an alluvial aquifer: A threedimensional N-dynamics model. Journal of Hydrology (New Zealand), 42, 145-162.

Widdowson, M.A. (1991). Comment on "An evaluation of mathematical models of the transport of biologically reacting solutes in saturated soils and aquifers" by Philippe Baveye and Albert Valocchi. Water Resources Research, 27(6), 1375-1378.

Woodward, S.J.R., Wöhling, T., Rajanayaka, C., and Wang, F. (2009). 3D modelling of nitrogen attenuation in coupled vadose zone-groundwater systems. In: The New Zealand Hydrological and Freshwater Sciences Societies Joint Conference, 23-27 November 2009, Whangarei, New Zealand.

Yeh, G.T., and Tripathi, V.S. (1989). A critical evaluation of recent developments in hydrogeochemical transport models of reactive multichemical components. Water Resources Research, 25(1), 93-108. 
Zhang, R., Huang, K., and Van Genuchten, M.T. (1993). An efficient Eulerian-Lagrangian method for solving solute transport problems in steady and transient flow fields. Water Resources Research, 29(12), 4131-4138.

\section{APPENDIX: ERRATA TO WANG ET AL. (2003)}

The FEMWATER-N flow-transport-reaction model discussed in this paper was first presented by Wang et al. (2003). Unfortunately, that paper contains several typographical errors, which we here take the opportunity to correct:

1. In equation (5), the second term on the right hand side should be ${ }^{L\left(c_{l}^{o}\right)}$.

2. In equation (6), the last term on the right hand side should be $-\mathfrak{R}_{\text {aer }}^{O}$.

3. In equation (7), the second term on the left hand side should be omitted.

4. In equation (8), the second term on the right hand side should be $-\mathfrak{R}_{\text {aer }}^{S}$.

5. In equation (9), the left hand side should be $\frac{\partial\left(\rho_{s} c_{s}^{s}\right)}{\partial t}$. This was not erroneous, but it is more consistent to express $c_{s}^{S}$ in mass units.

6. In equation 10, the second term on the left hand side should be omitted.

7. In table 2 , the distribution coefficients should be 5.01 water $/ \mathrm{kg}$ solid.

8. In table 2 , the second half rate concentration should be $K_{\min }^{O}$.

Additional parameters are also required in equations (13 a)-(13 f) to ensure dimensional consistency. These equations should be rewritten as

$$
\begin{aligned}
& \mathfrak{R}_{b \rightarrow l}^{\gamma}=\alpha^{\gamma} \theta_{b}\left(c_{b}^{\gamma}-K_{b \rightarrow l}^{\gamma} c_{l}^{\gamma}\right) \\
& \mathfrak{R}_{s \rightarrow l}^{S}=\beta \rho_{s}\left(c_{s}^{S}-K_{s \rightarrow l}^{S} c_{l}^{S}\right)
\end{aligned}
$$

where $K_{b \rightarrow l}^{\gamma}$ are equilibrium constants (similar to distribution coefficients) which have units $\mathrm{L}^{3}$ liquid phase $/ \mathrm{L}^{3}$ bio phase, and $K_{s \rightarrow l}^{S}$ is an equilibrium constant which has units $\mathrm{L}^{3}$ liquid phase/M solid phase. In the simulations carried out to date, the $K_{b \rightarrow l}^{\gamma}$ constants have been assumed to have a value of $1 \mathrm{~L}^{3}$ liquid $/ \mathrm{L}^{3}$ bio phase. Note that this commonly used kinetic sorption model contains the assumption that water content does not affect sorption-desorption rate (Severino et al., 2006).

Species transformations take place in the bio phase. To make this explicit, equations 17 to 20 should be rewritten with $V_{\min }^{\max } \theta_{b}, V_{n i t}^{\max } \theta_{b}, V_{a e r}^{\max } \theta_{b}$, and $V_{d e n}^{\max } \theta_{b}$ as their maximum rates respectively (i.e. the $V^{\max }$ constants are defined on the volume of the bio phase instead of on the volume of the medium). 